\title{
In vivo EPR on spin labeled colicin A reveals an oligomeric assembly of the pore-forming domain in $E$. coli membranes $\dagger$
}

2015, 17, 4875

Received 4th December 2014

Accepted 16th January 2015

DOI: $10.1039 / \mathrm{c} 4 \mathrm{cp} 05638 \mathrm{~h}$

www.rsc.org/pccp

\author{
S. Dunkel, L. P. Pulagam, $\neq$ H.-J. Steinhoff* and J. P. Klare*
}

\begin{abstract}
We report on the application of site-directed spin labeling (SDSL) and electron paramagnetic resonance (EPR) spectroscopy to study possible oligomerization of the bacterial toxin colicin A (ColA) upon membrane insertion in vitro and in vivo. We applied SDSL-EPR protocols and optimized experimental conditions to perform continuous wave EPR experiments and double electron-electron resonance distance measurements on intact Escherichia coli cells interacting with nitroxide spin-labeled ColA. Our data suggest that ColA forms dimers upon membrane insertion, thus explaining previously reported pore diameters of about $1 \mathrm{~nm}$, which are unlikely to be formed by a single colicin A monomer.
\end{abstract}

Colicin A is a water-soluble pore-forming protein toxin of $63 \mathrm{kDa}$ size produced by $E$. coli and lethal for related strains of the Enterobacteriaceae family, which are not protected by an immunity protein. Toxicity is achieved by inserting helical segments of the C-terminal pore-forming subdomain into the cytoplasmic membrane to form voltage-dependent ion channels, ${ }^{1-3}$ which lead to depolarization of the cell membrane followed by depletion of the intracellular ATP levels and finally to cell death. ${ }^{4}$ The receptor binding and translocation domains of the three domain protein are required for binding to the vitamin B12 receptor (BtuB) in the outer membrane and translocation of the pore-forming domain with the aid of OmpF, TolB, TolA, TolQ and TolR proteins. ${ }^{5,6}$

The soluble structure of the pore-forming domain (pfd) (Fig. 1, top panel) is characterized by two hydrophobic helices (hydrophobic hairpin H8-H9) that are surrounded by eight amphipathic $\alpha$-helices (H1-H7, H10). ${ }^{7}$ The same structural motif is conserved in several other pore-forming members of the colicin family and is also present in the channel-forming domains of other

Department of Physics, University of Osnabrück, Barbarastr. 7, 49076 Osnabrück, Germany. E-mail: jklare@uos.de; Fax: +49 541969 2656; Tel: +49 5419692664 $\dagger$ Electronic supplementary information (ESI) available: Experimental procedures and DEER results for soluble ColA-A192R1 and for a dilution series with different ColA : lipid ratios. See DOI: 10.1039/c4cp05638h

¥ Present address: Case Western Reserve University, 10900 Euclid Avenue, Cleveland, OH 44106, USA.
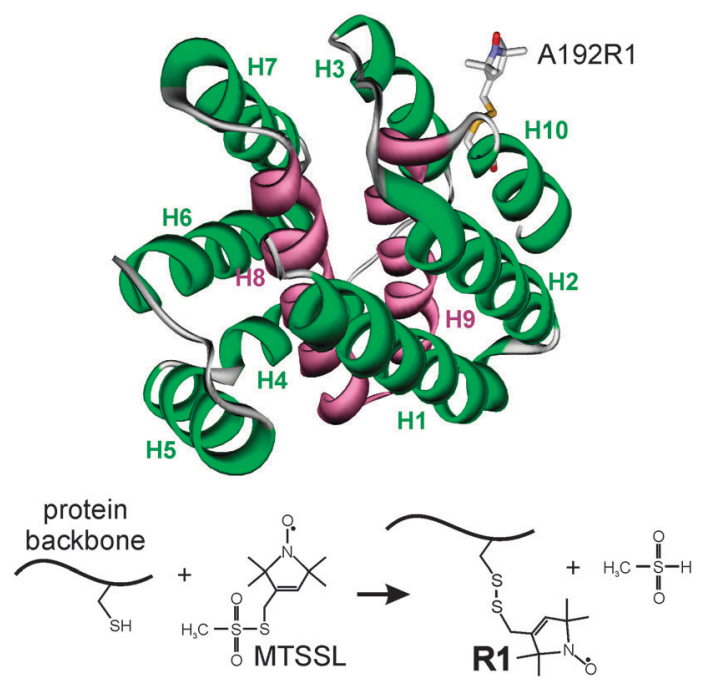

Fig. 1 Top panel: crystal structure of the ColA pfd (pdb: 1COL7), with a spin label side chain attached at position A192. Amphipathic helices are colored in green and the hydrophobic hairpin is shown in magenta. Bottom panel: site directed spin labeling. After site-directed mutagenesis to replace the residue of interest by cysteine, reaction of the methanethiosulfonate spin label (MTSSL) with the thiol of the cysteine yields the spin label side R1.

toxins like diphtheria toxin, exotoxin A, and the Bcl-2 family of pro- and anti-apoptotic proteins like Bax, Bak or Bcl-xL. ${ }^{8}$ A high-resolution structure of the membrane-inserted form of a pore-forming colicin is still not available, but solid-state $\mathrm{NMR}^{9,10}$ biochemical $^{11,12}$ and SDSL-EPR investigations ${ }^{13,14}$ have suggested that the initial membrane-bound state of the pfd exists as a two-dimensional helical array of the amphipathic helices spread across the membrane surface, with the hydrophobic hairpin embedded in the bilayer, supporting the 'umbrella' model for the closed-channel state. ${ }^{7}$

Since decades the exact conformation of the membranebound closed channel state of pore-forming colicins like ColA or Col Ia, and the mechanism of the membrane-potential induced pore-formation are strongly debated since some experimental observations appear contradictory. Besides an alternative model 
for the closed-channel state - the "penknife" $\operatorname{model}^{7,15}$ in which the hydrophobic hairpin is also residing in the headgroup region of the bilayer - several models exist for the formation of the open-channel state by translocation of specific helical segments across the membrane. ${ }^{6}$ Some of the most controversial experimental observations being relevant in this context concern the properties of the open pore and how many pfds are necessary to form the pore. Multiple lines of evidence exist affirming that colicin channels are monomers. ${ }^{16}$ Nevertheless, the size of the pores appear to be too large to be formed by a single pfd, as the passage of ions as large as tetraethyl ammonium indicate a pore diameter of at least $1 \mathrm{~nm} .^{6}$ Thus, oligomerization of pfds in the membrane to form such large pores, like it is known but yet not fully understood for the closely related Bcl-2 family, appears to be inevitable. Nevertheless, to date only a single (Cryo-EM) study on Col Ia provided direct experimental evidence for the formation of colicin oligomers in a lipid bilayer, even though under highly unphysiological conditions. ${ }^{17}$

Site-directed spin labeling of proteins ${ }^{18,19}$ combined with EPR spectroscopy is a valuable tool to assess the structural properties of membrane proteins and membrane protein complexes ${ }^{18-21}$ and can - via inter spin distance measurements either with continuous wave (cw) or pulsed experiments - also report on the oligomeric state of the system under investigation. ${ }^{22-25}$ Nevertheless, although SDSL-EPR is a well established method for in vitro investigations under close to physiological conditions, in vivo EPR with spin labeled proteins in living cells is challenging due to several complications. Besides the usually low protein concentrations present in cells, foremost the necessity to perform a labeling reaction in the often reducing and chemically complex cellular environment - anticipating the commonly applied labeling of sitespecifically introduced cysteine side-chains - and the requirement of reduction-resistant spin labels, are problems that are yet only partly solved. Nevertheless, recent studies could demonstrate the general applicability of SDLS-EPR in vivo. ${ }^{26,27}$ For example, although nitroxides are readily reduced under in vivo conditions, ${ }^{28-30}$ their use for in vivo spin labeling of proteins in E. coli has been recently reported. ${ }^{31}$

In the case of ColA, labeling can be carried out with purified proteins in vitro and the labeled protein can be incubated with $E$. coli cells, thus minimizing the harmful influence of the cellular environment on the label side chain. We chose an E. coli strain (Origami ${ }^{\circledR}$ ) being deficient of active thioredoxin reductase and glutathione reductase to further alleviate the reductive conditions in the cells, as it has been suggested that nitroxide deactivation in vivo is an enzymatic or enzyme-mediated reduction process. ${ }^{32}$ For our investigations, we labeled ColA with MTSSL at position 192, located on helix H10 (Fig. 1). A viability test with $E$. coli cells (see ESI $\dagger$ Materials and methods) confirmed that the toxic activity of ColA-A192R1 is not reduced compared to the wt protein.

Cw EPR spectra at room temperature have been recorded for ColA-A192R1 in the soluble state, reconstituted in liposomes prepared from $E$. coli polar lipids, and incubated with live $E$. coli Origami cells (Fig. 2). When comparing the EPR spectra of soluble ColA with liposome-reconstituted and cell-bound ColA,

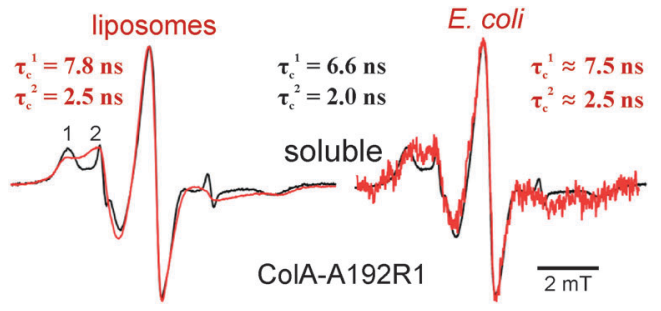

Fig. 2 Room temperature cW EPR spectra recorded from ColA-A192R1 in solution (black), reconstituted in E. coli polar lipid liposomes (red, left panel) and from live E. coli Origami cells incubated with ColA-A192R1 (red, right panel). Rotational correlation times $\tau_{\mathrm{c}}$ for the two spectral components 1 and 2 (indicated in the left panel) obtained from spectra simulations are given. The sharp EPR lines in the low-field and high-field regions of the spectrum for soluble ColA are due to a small amount of unbound spin label in the sample.

the observed spectral changes clearly indicate a conformational change upon interaction of ColA with artificial and native membranes. Although the spectra recorded in vitro and in vivo are not identical, the overall mobility changes are comparable, indicating similar conformations of the membrane-bound state. This is also reflected in the rotational correlation times $\tau_{\mathrm{c}}$ determined from simulations of the EPR spectra (given in Fig. 2) that all show the presence of two spectral components characterized by different spin label mobilities. Such multicomponent spectra can arise either from structural constraints in the microenvironment of the spin label, leading to different stable spin label side chain conformations or different protein conformations in equilibrium. ${ }^{33,34}$

Double electron-electron resonance (DEER) $(a k a$ PELDOR $=$ pulsed electron double resonance) inter-spin distance measurements performed on proteoliposomes containing ColA-A192R1 (Fig. 3, left panel) reveal a dipolar modulation of the signal that is not observed for the protein in solution (Fig. S1, ESI $\dagger$ ), indicating that the ColA molecules carrying a single spin label side chain interact in liposomes, forming dimers or higher oligomers. For data analysis, a single Gaussian distance distribution was assumed to fit the experimental data, yielding a mean distance of $2.9 \mathrm{~nm}$ and a distance distribution width (full width at half maximum, FWHM) of $0.7 \mathrm{~nm}$.

The modulation depths of the DEER signal, reporting on the number of interacting spin labels, yields a value of $\sim 1.8$ for ColA-A192R1 reconstituted into liposomes. Taking the labeling efficiency of almost $100 \%$ into account, this would point to a dimeric assembly. Nevertheless, it cannot be excluded that higher oligomers are formed as the number of interacting spins depends on the labeling efficiency, which might be lower than $100 \%$ due to partial reduction, the number of protomers in the oligomer and on the dissociation constant for ColA oligomerization in membranes, of which the latter is unknown.

Reconstitution of spin-labeled ColA into liposomes for the EPR experiments was carried out with a high protein : lipid ratio of $\sim 1: 500(\mathrm{M} / \mathrm{M})$ to maximize the EPR signal strength. In order to test if such high protein concentrations in liposomes might artificially induce unspecific oligomerization, we prepared a dilution series with reconstitutions carried out with different protein:lipid ratios, ranging from $1: 500$ to $1: 5000(\mathrm{M} / \mathrm{M})$. 

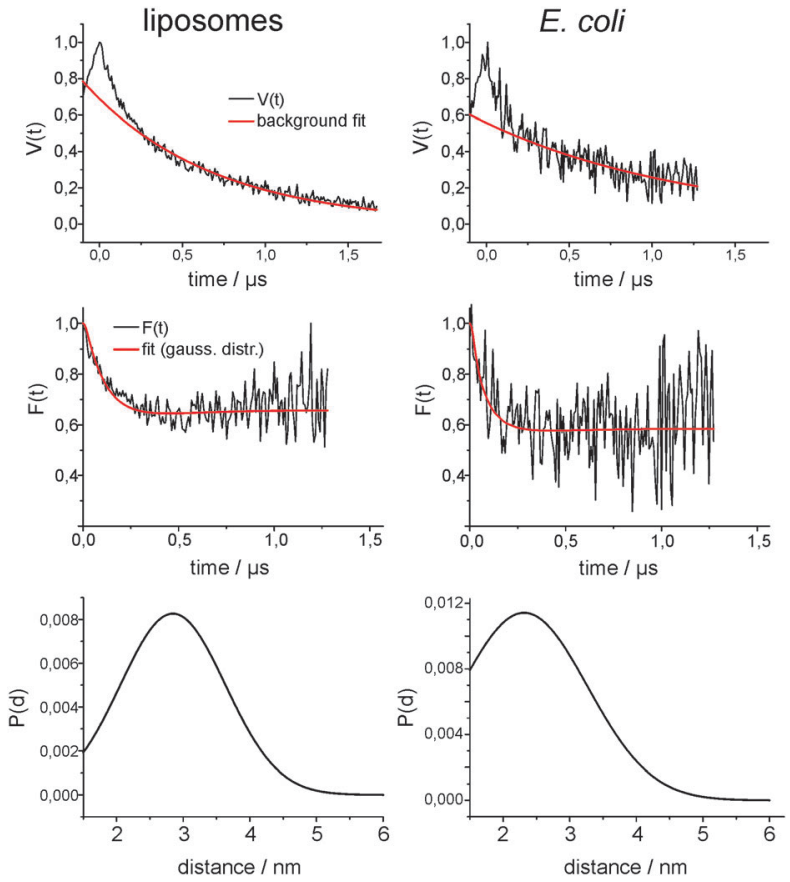

Fig. 3 Left panel: DEER measurements performed on proteoliposomes containing ColA-A192R1. Right panel: DEER measurements with E. coli cells treated with spin labeled ColA-A192R1. In each panel the upper row shows the raw DEER data, $V(t)$, with the corresponding background fit (red), leading to the form factors, $F(t)$, shown in the middle row (with fits (red) obtained by assuming a single gaussian distance distribution). The bottom row shows the resulting distance distributions, $P(t)$. Details of data analysis procedures are given in the ESI. $\dagger$

DEER measurements on these samples (Fig. S2, ESI $\dagger$ ) reveal that oligomerization is detectable already at a protein:lipid ratio of $1: 2500$, providing an average membrane area of $\sim 30 \times 30 \mathrm{~nm} \mathrm{ColA}^{-1}$ molecule. ${ }^{35}$ The DEER modulation depths increase with increasing protein:lipid ratio, indicating a high dissociation constant for ColA oligomerization in membranes. For the protein:lipid ratios tested the number of interacting spins determined from the DEER data does not exceed 2, indicating dimerization of the protein.

The cw EPR spectra recorded from proteoliposomes and from live E. coli cells incubated with spin-labeled ColA (Fig. 2) already indicated similar conformational changes upon interaction with artificial and native membranes, suggesting that oligomerization might also take place in vivo. To test this, we performed DEER measurements with $E$. coli cells treated for 2 min with ColA-A192R1 and washed from unbound ColA prior to freezing in liquid nitrogen (Fig. 3, right panel). Despite the significantly lower signal-to-noise ratio obtained for this sample, the experiment unambiguously reveals a dipolar interaction also in vivo. Analysis of the data in terms of a distance distribution yields a mean distance of $2.6 \pm 0.7 \mathrm{~nm}$, comparable to the results obtained for proteoliposomes. Interestingly, the modulation depth in vivo appears to be higher $(\approx 2)$ compared to that obtained for the in vitro sample. This observation suggests a higher propensity for ColA to form oligomers under physiological conditions, thus strongly supporting the physiological relevance of oligomerization for pore formation. Based on the similarity to Bcl-2 family proteins that have been shown to form homo- and heterodimers and most likely also larger assemblies thereof, ${ }^{36}$ a ColA dimer appears to be the most likely candidate. Furthermore, in the cryo-electron microscopy study on Colicin Ia by Greig et $a l^{17}$ electron density maps show oligomeric assemblies of membrane-inserted pfds, that can easily be interpreted as trimers of dimers, based on the apparent $c_{3}$ symmetry and the estimated molecular mass of the objects, ${ }^{17}$ thus further supporting the assumption that ColA pfds might form dimers in the membrane. Indeed, assuming that in addition to the hydrophobic hairpin two or more amphipathic helices become transmembrane in the open-channel state induced by the membrane potential, ${ }^{6}$ a total number of 8-10 membrane-spanning helices should suffice to form a pore of about $1 \mathrm{~nm}$ diameter.

We demonstrate by applying SDSL-EPR that the pore-forming domain of colicin A forms oligomers in artificial membranes as well as in the membrane of live E. coli cells, most likely in the form of dimers. The peculiarities of this system render standard site-directed spin labeling approaches sufficient for in vivo investigations, and numerous applications of SDSL EPR can be envisioned that similarly take advantage of the system's specific properties (e.g. for the investigation of other pore-forming toxins, cell-penetrating peptides, or molecules that are imported into the cell or interact with components at the cell surface). Current progress in the development of alternative SDSL approaches and reduction resistant spin labels will further broaden the applicability of SDSL-EPR in vivo.

\section{Acknowledgements}

We acknowledge the DFG priority program SPP1601 for financial support.

\section{Notes and references}

1 J.-P. Bourdineaud, P. Boulanger, C. Lazdunski and L. Letellier, Proc. Natl. Acad. Sci. U. S. A., 1990, 87, 1037.

2 W. A. Cramer, F. E. Cohen, A. R. Merrill and H. Y. Song, Mol. Microbiol., 1990, 4, 519.

3 J. H. Lakey, F. Gisou van der Goot and F. Pattus, Toxicology, 1994, 87, 85.

4 W. A. Cramer, J. B. Heymann, S. L. Schendel, B. N. Deriy, F. S. Cohen, P. A. Elkins and C. V. Stauffacher, Annu. Rev. Biophys. Biomol. Struct., 1995, 24, 611.

5 J. C. Lazzaroni, J. F. Dubuisson and A. Vianney, Biochimie, 2002, 84, 391.

6 E. Cascales, S. K. Buchanan, D. Duche, C. Kleanthous, R. P. Lloubes, K. Postle, M. Riley, S. Slatin and D. Cavard, Microbiol. Mol. Biol. Rev., 2007, 71, 158.

7 M. W. Parker, J. P. M. Postma, F. Pattus, A. D. Tucker and D. J. Tsernoglou, J. Mol. Biol., 1992, 224, 639.

8 M. W. Parker and F. Pattus, Trends Biochem. Sci., 1993, 18, 391.

9 X. L. Yao and M. Hong, Biochemistry, 2006, 45, 289. 
10 Z. Wei, D. White, J. Wang, A. A. Musse and A. R. Merrill, Biochemistry, 2007, 46, 6074.

11 P. K. Kienker, X. Qiu, S. L. Slatin, A. Finkelstein and K. S. J. Jakes, J. Membr. Biol., 1997, 157, 27.

12 D. Duché, M. W. Parker, J. M. González-Mañas, F. Pattus and D. Baty, Biol. Chem., 1994, 269, 6332.

13 P. V. Padmavathi and H.-J. Steinhoff, J. Mol. Biol., 2008, 378, 204.

14 S. Böhme, P. V. Padmavathi, J. Holterhues, F. Ouchni, J. P. Klare and H.-J. Steinhoff, Phys. Chem. Chem. Phys, 2009, 11, 6770.

15 J. H. Lakey, D. Duché, J. M. González-Mañas, D. Baty and F. Pattus, J. Mol. Biol., 1993, 230, 1055.

16 S. Slatin and P. Kienker, in Pore forming peptides and protein toxins, ed. G. Menestrina, Taylor and Francis, London, 2003, p. 102.

17 S. L. Greig, M. Radjainia and A. K. Mitra, J. Biol. Chem., 2009, 284, 16126.

18 C. Altenbach, S. L. Flitsch, H. G. Khorana and W. L. Hubbell, Biochemistry, 1989, 28, 7806.

19 J. P. Klare and H.-J. Steinhoff, Photosynth. Res., 2009, 102, 377.

20 E. Bordignon and H.-J. Steinhoff, in ESR Spectroscopy in Membrane Biophysics, ed. M. A. Hemminga and L. J. Berliner, Springer, New York, 2007, p. 129.

21 B. Jagannathan, S. Dekat, J. H. Golbeck and K. V. Lakshmi, Biochemistry, 2010, 49, 2398.

22 B. E. Bode, D. Margraf, J. Plackmeyer, G. Dürner, T. F. Prisner and O. Schiemann, J. Am. Chem. Soc., 2007, 129, 6736.
23 S. Meyer, S. Böhme, A. Krüger, H.-J. Steinhoff, J. P. Klare and A. Wittinghofer, PLoS Biol., 2009, 7, e1000212.

24 J. E. Banham, C. R. Timmel, R. J. Abbott, S. M. Lea and G. Jeschke, Angew. Chem., Int. Ed., 2006, 45, 1058.

25 T. Vöpel, C. S. Hengstenberg, T. O. Peulen, Y. Ajaj, C. A. M. Seidel, C. Herrmann and J. P. Klare, Biochemistry, 2014, 53, 4590.

26 M. Azarkh, V. Singh, O. Okle, I. T. Seemann, D. R. Dietrich, J. S. Hartig and M. Drescher, Nat. Protoc., 2013, 8, 131.

27 M. J. Swamy, L. Ciani, M. Ge, A. K. Smith, D. Holowka, B. Baird and J. H. Freed, Biophys. J., 2006, 90, 4452.

28 L. Krstic, R. Hansel, O. Romainczyk, J. W. Engels, V. Dotsch and T. F. Prisner, Angew. Chem., Int. Ed., 2011, 50, 5070.

29 W. R. Couet, R. C. Brasch, G. Sosnovsky and T. N. Tozer, Magn. Reson. Imaging, 1985, 3, 83.

30 S. Belkin, R. J. Mehlhorn, K. Hideg, O. Hankovsky and L. Packer, Arch. Biochem. Biophys., 1987, 256, 232.

31 M. J. Schmidt, J. Borbas, M. Drescher and D. J. Summerer, J. Am. Chem. Soc., 2014, 136, 1238.

32 M. Azarkh, O. Okle, P. Eyring, D. R. Dietrich and M. Drescher, J. Magn. Reson., 2011, 212, 450.

33 W. L. Hubbell, D. S. Cafiso and C. Altenbach, Nat. Struct. Biol., 2000, 7, 735.

34 R. Langen, K. J. Oh, D. Cascio and W. L. Hubbell, Biochemistry, 2000, 39, 8396.

35 V. Luzatti, Biological Membranes, Academic Press, New York, 1986.

36 G. Dewson, T. Kratina, P. Czabotar, C. L. Day, J. M. Adams and R. M. Kluck, Mol. Cell, 2009, 36, 696. 